\title{
A Comprehensive Review of Sarcoidosis Diagnosis and Monitoring for the Pulmonologist
}

\author{
Andrea S. Melani (D) - Albano Simona · Martina Armati · \\ Miriana d'Alessandro · Elena Bargagli
}

Received: January 30, 2021 / Accepted: May 17, 2021 / Published online: June 6, 2021

(C) The Author(s) 2021

\begin{abstract}
Sarcoidosis is a systemic granulomatous disease with heterogenous clinical manifestations. Here we review the diagnosis of sarcoidosis and propose a clinically feasible diagnostic work-up and monitoring protocol. As sarcoidosis is a
\end{abstract}

A. S. Melani $(\square) \cdot$ A. Simona

UOS Pneumologia/UTIP, Dip. Scienze Mediche, Chirurgiche E Neuroscienze, Policlinico Le Scotte Viale Bracci, Azienda Ospedaliera Senese, 53100 Siena, Italy

e-mail: a.melani@ao-siena.toscana.it

\section{A. Simona}

e-mail: s.albano@gmail.com

M. Armati · M. d'Alessandro · E. Bargagli

UOC Malattie Respiratorie, Dip. Scienze Mediche, Chirurgiche E Neuroscienze Università Di Siena

Policlinico "Le Scotte", Siena, Italy

M. Armati

e-mail: martina.armati@gmail.com

M. d'Alessandro · E. Bargagli

UOC Malattie Respiratorie, Immunoallergology, Rare Respiratory Diseases and Lung Transplant Laboratory, Dip. Scienze Mediche, Chirurgiche E Neuroscienze Università Di Siena Policlinico "Le Scotte", Siena, Italy

M. d'Alessandro

e-mail: dalessandro.miriana@gmail.com

E. Bargagli

e-mail: bargagli2@gmail.com systemic disease, a multidisciplinary approach is recommended for best outcomes. However, since the lungs are frequently involved, the pulmonologist is often the referral physician for diagnosis and management. When sarcoidosis is suspected, diagnosis needs to be confirmed and organ involvement/impairment assessed. This process is also required to establish whether the patient is likely to benefit from treatment, as many cases of sarcoidosis are selflimited and remit spontaneously. Whether or not treatment is started, effective regular followup is necessary to monitor changes in the disease, including extension, progression, remissions, flare-ups, and complications.

Keywords: Sarcoidosis; Diagnosis; Monitoring; Biomarkers; Clinical evaluation 


\section{Key Summary Points}

Sarcoidosis is a relatively uncommon disease with heterogeneous onset and manifestations, which are often shared with other more common diseases. Early diagnosis is challenging, and thus diagnosis is often delayed.

The diagnosis of sarcoidosis relies on a compatible clinical and radiological picture, biopsy evidence of non-caseating, non-necrotizing granulomas, and exclusion of other similar diseases. Alternative diagnoses are always possible and should be borne in mind.

As sarcoidosis is a systemic disease, a multidisciplinary approach is suggested for best outcomes. However, the pulmonologist usually has a key role in diagnosis and management, because the lungs and thoracic lymph nodes are the organs most commonly involved.

If sarcoidosis is suspected, an extensive work-up is required to establish organ involvement. Close follow-up is also necessary, as sarcoidosis is a chronic disease with an unpredictable course.

There is need for safe, easy, and reliable biomarkers to establish organ involvement and prognosis at the individual level.

\section{DIGITAL FEATURES}

This article is published with digital features, including a summary slide, to facilitate understanding of the article. To view digital features for this article go to https://doi.org/10.6084/ m9.figshare.14602320.

\section{INTRODUCTION}

Sarcoidosis is a systemic inflammatory disease of unknown aetiology, characterized histologically by non-necrotizing granulomatous inflammation, mostly affecting the lungs and thoracic lymph nodes. Here we review results from studies on sarcoidosis found in PubMed searches. The search was performed in the electronic databases of MEDLINE for relevant literature using a combination of the terms "sarcoidosis" [MeSH] OR "sarcoid" *[tiab] AND "autoimmune diseases" "[MeSH] up to 31 December 2020. The search yielded more than 3800 hits and selected references considered as pertinent, mainly including papers presented in full text and in English. Retrieved references were hand-searched for additional papers on the topic. This article is based on previously conducted studies and does not contain any new studies with human participants or animals performed by any of the authors. The aim of the study is to propose emerging methods for the diagnosis and monitoring of sarcoidosis.

\section{EPIDEMIOLOGY}

Sarcoidosis is observed worldwide, although with geographical, ethnic, and environmental variations. It mainly affects people in the third and fourth decades of life, but may also occur in children and the elderly. The real incidence and prevalence of sarcoidosis worldwide is difficult to determine, as many patients are asymptomatic. In terms of frequency and severity, it has a higher prevalence in individuals of Nordic European descent, African Americans, and economically disadvantaged populations (about $60 / 100,000)$ than in southern European countries, including Italy $(<10 / 100,000)[1,2]$. The disease is more common in females than males, non-smokers than smokers, and in certain races such as African Americans, whereas children are seldom affected [2]. Mortality is estimated at about $2-4 \%$ of cases, generally related to lung fibrosis and respiratory failure, but rarely to heart involvement (sudden cardiac death) [3]. 


\section{AETIOPATHOGENESIS}

Sarcoidosis is characterized by a T-helper response in which CD4 lymphocytes and activated macrophages accumulate in affected organs, resulting in the formation of granulomas. Sarcoidosis is believed to develop in patients with genetic susceptibility after exposure to as yet unidentified antigens. These antigens are presumably sensed and processed by macrophages, but not fully cleared for unknown reasons, creating engulfment. This activates undifferentiated histiocytes into M2 phenotype macrophages, expansion and polarization of CD4+ lymphocytes towards Th1, Th17, and Th17.1 cells, impairment of T regulatory cell (Treg) function, and development of sarcoid granulomas with inflammation that releases a broad spectrum of mediators, including cytokines, chemokines, and oxygen radicals that are involved in aetiopathogenesis [4]. Familiar forms of sarcoidosis are reported in less than $10 \%$ of cases. The disease is polygenic, associated with specific human leukocyte antigens (class I: HLA-A, B, C; class II: HLA-DP, DQ, DR) $[2,5,6]$. Whether these not fully efficient responses are primary or secondary to local persistent antigen stimulation is unclear. The phenotype involves an interaction between patient genotype and environmental factors.

Although the triggers for sarcoidosis are still unclear, certain environmental factors have been associated with the disease. The ACCESS study (A Case Control Etiologic Study of Sarcoidosis), which recruited more than 700 incident cases and matched them with controls, did not show a clear cause, but suggested specific occupations and exposures that were more prevalent in patients with sarcoidosis. These included agriculture, health care, bird breeding, automotive industry, and middle/high school teaching. Exposures included insecticides, mildew, mould, musty odours, and home central air conditioning [7]. Potential triggers are $M y$ cobacterium tuberculosis and Cutibacterium acnes (Propionibacterium acnes), but sarcoidosis is not an active infectious disease [8]. Smokers have a lower incidence of sarcoidosis, and nicotine has been posited to have a protective role in sarcoidosis [9].

\section{CLINICAL MANIFESTATIONS}

The clinical manifestations of sarcoidosis are heterogeneous and depend on the organ(s) involved and the degree of involvement. The frequency and main manifestations of organ involvement in four large case series are summarized in Table 1 [10-13]. Although any part of the body may be involved, the lungs and thoracic lymph nodes are by far the most commonly affected organs. In a retrospective series of 1686 patients in the United States with biopsy-proven sarcoidosis, extrathoracic lesions alone were only observed in $8.3 \%$ of cases [14], although the frequency of organ involvement was dependent on the investigation method used. Many patients with sarcoidosis, symptomatic at baseline, have respiratory symptoms such as cough and/or exertional dyspnoea. Generalized complaints such as fever, night sweats, and fatigue are also described in up to three-quarters of cases [10-13], possibly due to systemic spillage of inflammatory mediators. Fatigue does not prevent physical activity, but may be highly disabling and have an adverse impact on quality of life [15]. Indeed, physical activity is reported to be significantly lower in patients with sarcoidosis than in healthy controls [16]. However, fatigue and other generalized symptoms should only be attributed to sarcoidosis when other causes, such as depression, adrenal and thyroid dysfunction, and sleep breathing disorders, have reasonably been excluded. Not only is onset of sarcoidosis highly variable, sometimes acute, sometimes quite insidious, but the disease course is also unpredictable, sometimes constantly subclinical, sometimes evolving, with very severe symptoms and impact on quality of life and prognosis.

\section{DIAGNOSIS}

Knowledge of the wide range of sarcoidosis onset and organ involvement is important to reduce delays in diagnosis and/or 
Table 1 Organ involvement and main clinical manifestations of sarcoidosis

\begin{tabular}{|c|c|c|c|c|c|}
\hline Reference no. & 5 & 6 & 7 & 8 & \\
\hline No cases & 736 & 1774 & 640 & 2163 & \\
\hline $\begin{array}{l}\text { Involved organ, } \% \\
\text { affected }\end{array}$ & & & & & Clinical characteristics \\
\hline Lungs & 95 & 89 & 91 & 93 & Cough, dyspnoea (mainly in stages 3 and 4) \\
\hline Liver & 11.5 & 20 & 18 & 5 & $\begin{array}{l}\text { Mostly asymptomatic, sometimes associated with increase in liver function } \\
\text { tests }\end{array}$ \\
\hline Spleen & 7 & 7 & 7.5 & 4 & Sometimes splenomegaly, seldom cytopenia \\
\hline $\begin{array}{l}\text { Eye (excluding } \\
\text { lacrimal glands) }\end{array}$ & 12 & 23 & 8 & 8 & Red, painful eye, photophobia, blurring, decreased vision \\
\hline Muscle & 0.4 & 1 & 1 & 7.5 & Muscle weakness, myalgia \\
\hline Joint and bone & 0.5 & 7 & 3 & 10 & $\begin{array}{l}\text { Arthralgia, arthritis, mostly acute, bilateral with functional impairment; } \\
\text { bone pain }\end{array}$ \\
\hline $\begin{array}{l}\text { Peripheral lymph } \\
\text { nodes }\end{array}$ & 15 & 12 & 18 & 11 & $\begin{array}{l}\text { Lymphadenopathy, mostly recognized by clinical examination in cervical } \\
\text { and supracervical areas }\end{array}$ \\
\hline Bone marrow & 4 & 8 & $<1$ & NA & Pancytopenia \\
\hline Skin & $16^{\mathrm{a}}$ & 32 & 21 & 16 & $\begin{array}{l}\text { A range of lesions, sometimes nonspecific, such as erythema nodosum, } \\
\text { often coexisting and even occurring in scars or tattoos; lupus pernio }\end{array}$ \\
\hline Nervous system & 5 & 9 & 9 & 3.4 & A range of clinical findings and severities \\
\hline Heart & 2 & 5 & 1 & 3 & $\begin{array}{l}\text { A range of clinical findings and severities, including cardiac failure and/or } \\
\text { arrhythmias }\end{array}$ \\
\hline Urogenital $^{\mathrm{b}}$ & 1 & 1 & 3 & 3.5 & Renal interstitial nephritis \\
\hline $\begin{array}{l}\text { Parotid and } \\
\text { lacrimal glands }\end{array}$ & 4 & 3 & 2 & 4 & Painless gland swelling, lacrimation, dry eye (in chronic variants) \\
\hline Upper airways & 3 & 1 & 3 & NA & Nodules, polyps, nasal obstruction \\
\hline Gastrointestinal & 0 & NA & 0 & 1 & Mostly asymptomatic \\
\hline
\end{tabular}

${ }^{a}$ Excluding erythema nodosum, ${ }^{b}$ excluding calcium disturbances and their consequences $N A$ not available

underdiagnosis. Cases with typical onset and symptoms can be diagnosed early and relatively accurately. These phenotypes include Löfgren syndrome with erythema nodosum (a painful red skin rash typically found on the anterior surface of the forearms), coupled with chest X-ray, evidence of bilateral hilar adenopathy, fever, arthritis or arthralgia; Heerfordt syndrome with fever, sometimes facial palsy, uveitis, parotitis, and mediastinal lymphadenopathy displaying imaging evidence of the panda and/or lambda sign [2]. However, many patients, perhaps about half, are asymptomatic at diagnosis, and many others have nonspecific respiratory or general symptoms shared with more common diseases. In these cases, the diagnosis of sarcoidosis is challenging, may be delayed by months to years, and may be suspected incidentally during a routine medical check-up for other reasons. There are 
recommendations with updated criteria for defining diagnosis and organ involvement in sarcoidosis [17-19], although applying them in real-world clinical settings is not always easy. The likelihood of organ involvement in sarcoidosis may be indicated as highly probable, probable, or possible [17, 18]. Many cases of sarcoidosis are discovered after a tissue biopsy finding of non-necrotizing, non-caseating granulomas. However, the finding does not suffice to diagnose sarcoidosis, as many other diseases can be associated with a similar feature of inflammation and need to be excluded (see Table 2). The diagnosis of sarcoidosis relies on three criteria: a compatible clinical and radiological presentation, biopsy evidence of noncaseating, non-necrotizing granulomas, and exclusion of other diseases with similar features (see Fig. 1) [17-19].

As any organ may be involved and offer adequate tissue specimens, if sarcoidosis is suspected, biopsies should preferably be obtained by the most safely accessible lesion. In a singlecentre series of unselected sarcoidosis patients, $23 \%$ were diagnosed without biopsy and $77 \%$ with biopsy. The specimen was obtained from the skin (23\%), muscle (12\%), peripheral lymph nodes $(11 \%)$, and transbronchial lymph nodes (15\%) [12]. As endoscopic techniques are improving, diagnosis by lung biopsies is increasing; in the British Thoracic Society (BTS) Sarcoidosis Registry, almost half of the biopsyproven diagnoses of sarcoidosis during the period from 2013 to 2017 were based on endo- or transbronchial tissue specimens [20]. In another UK single-centre series, 177 out of 503 patients with sarcoidosis had biopsy-confirmed diagnosis based on transbronchial or endobronchial specimens [21]. Since sarcoidosis is a systemic disease, once it is suspected or diagnosed, exhaustive study is required to define organ involvement [22]; our proposal is illustrated in Fig. 1.

\section{IMAGING METHODS}

Chest X-ray is the traditional imaging technique for detecting and classifying thoracic sarcoidosis, as shown in Table 3 [23]. Although
Table 2 Main diseases simulating pulmonary sarcoidosis requiring differential diagnosis

Foreign body granulomatosis (aspiration or intravenous injection of foreign materials)

Hypersensitivity pneumonia

Connective tissue diseases

Pneumoconioses (aluminum, beryllium, cobalt, talc, titanium, zirconium)

Common variable immune deficiency

Bacterial disease (brucellosis, leprosy, bartonellosis, tularaemia, cat scratch disease, Whipple's disease, nontuberculous mycobacteria, tuberculosis)

Fungal disease (aspergillosis, blastomycosis, coccidioidomycosis, cryptococcosis, histoplasmosis, Pneumocystis jirovecii)

Parasitic disease (echinococcosis, leishmaniasis, schistosomiasis, toxoplasmosis)

Viral disease (human immunodeficiency virus)

Bronchocentric granulomatosis, necrotizing sarcoid granulomatosis, inflammatory bowel disease

Eosinophilic granulomatosis, pulmonary Langerhans cell histiocytosis

Lymphocytic interstitial pneumonia, desquamative interstitial pneumonia

Granulomatous histiocytic necrotizing lymphadenitis (Kikuchi's disease)

Sarcoid-like lesions associated with past or concomitant carcinoma, lymphomas, or drugs such as immune checkpoint inhibitors, TNF-antagonists, interferons and mainly the alpha moiety, BRAF and ALK inhibitors, antiretroviral therapy, nitrofurantoin, quetiapine, thalidomide, etretinate, adjuvants such as silicone, hyaluronic acid, mineral oil

Crohn's disease

Vasculitis, (Churg-Strauss syndrome), granulomatosis with polyangiitis

Blau syndrome 


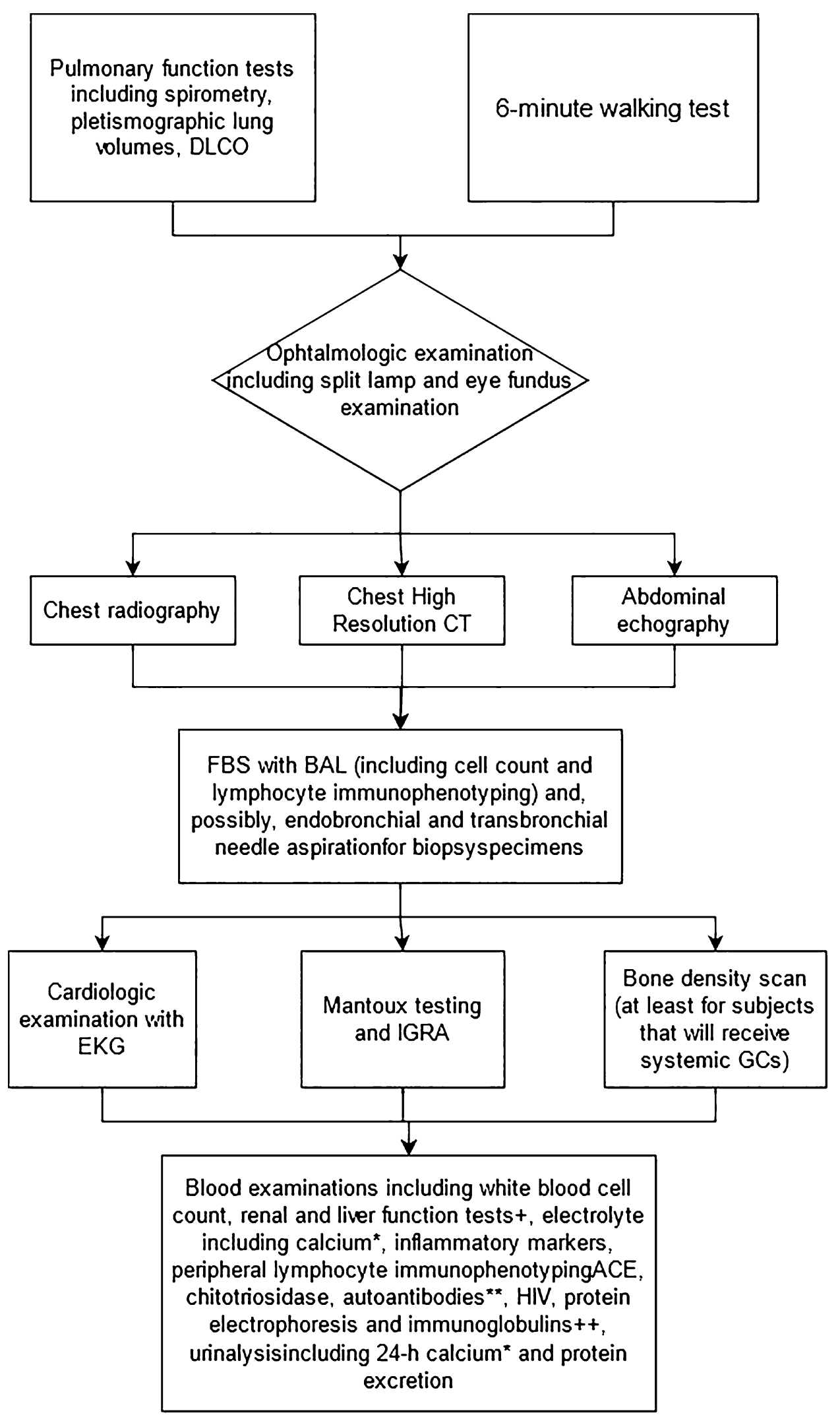


4Fig. 1 Suggested work-up in cases of suspected sarcoidosis. IGRA is usually preferred to check for latent tuberculosis, since sarcoidosis patients are often anergic to the intradermal Mantoux test ${ }^{+}$Elevated serum renal and liver function tests may be common in sarcoidosis involvement even in the absence of other signs and/or symptoms. ${ }^{*} 25$ Hydroxyvitamin D3, 1,25-hydroxyvitamin D3, and parathyroid hormone should be measured in patients with hypercalcaemia and/or hypercalciuria to assess the degree and the cause of calcium dysregulation; hypercalcaemia with low parathyroid hormone levels and normal or low 25-hydroxyvitamin D3 levels suggests sarcoidosis. ${ }^{* *} \mathrm{Au}$ toantibodies are typically negative (seldom positive at low titers) in sarcoidosis, while lymphopenia may sometimes be observed. ${ }^{++}$There is often a polyclonal hypergammaglobulinaemia, but never a low blood level of gamma globulinsDLco diffusing capacity of the lung for carbon monoxide, $C T$ computed tomography, $F B S$ fibrobronchoscopy, $B A L$ bronchoalveolar lavage, ECG electrocardiogram, IGRA Interferon- $\gamma$ release assay, $H I V$ human immunodeficiency virus, $A C E$ angiotensin-converting enzyme

rough and limited by inter-observer variability, $\mathrm{X}$-ray staging has prognostic relevance, as the likelihood of spontaneous remission without treatment decreases with increasing stage. In the multicentre US-based ACCESS study performed within 6 months from diagnosis, $15 \%$ of cases had a pattern suggesting stage 3 or 4 [10].

Chest computed tomography (CT) scans are often used to study lung lesions in greater detail or in patients with persistent respiratory signs and symptoms. The most common lung

Table 3 Scadding chest X-ray stages of sarcoidosis

\begin{tabular}{ll}
\hline Stage & X-ray description \\
\hline 0 & No chest abnormalities \\
1 & Bilateral hilar lymphadenopathy without \\
& parenchymal infiltrates \\
2 & Bilateral hilar lymphadenopathy and pulmonary \\
& infiltrates \\
3 & Pulmonary infiltrates without bilateral hilar \\
& lymphadenopathy \\
4 & Pulmonary fibrosis or lung conglomerate opacities \\
\hline
\end{tabular}

findings in sarcoidosis are bilateral nodules (due to sarcoid granulomas that aggregate into macroscopic lesions) with peribronchovascular distribution in the upper and middle lobes, sometimes forming mass-like perihilar consolidations. Scarring and air trapping may occur, usually distributed centrally along bronchovascular bundles, sometimes with bronchiectasis. Honeycombing, pleural involvement, extensive ground glass opacities (>50\%), and basilar involvement are not typical of sarcoidosis. Thoracic lymphadenopathy, often bilateral and symmetrical, is a common finding in up to $90 \%$ of affected patients [24]. The extent of fibrosis and the ratio of main pulmonary artery diameter to ascending aorta diameter are reported to have prognostic value [21].

Positron emission tomography with ${ }^{18} \mathrm{~F}$-labelled fluorodeoxyglucose (FDG), usually coupled with perfusion scans and computed tomography (FDG PET/CT), and magnetic resonance imaging (MRI) are increasingly performed, though not yet routine examinations in the work-up of sarcoidosis. They should be performed at specialized centres familiar with sarcoidosis [25-27]. FGD PET/CT is more sensitive than traditional gallium-67 scans in detecting active sarcoidosis lesions, but cannot diagnose sarcoidosis alone, because a positive finding may be the result of malignancy or other inflammatory conditions. FGD PET/CT is indicated when active sarcoid involvement is suspected in relatively inaccessible areas such as the heart, brain, or bone, eventually as a guide to indicate diagnostic biopsy sites; in symptomatic patients when conventional examinations give inconclusive indications regarding the need for immunosuppressive treatment; and in near-end-stage sarcoidosis lesions for monitoring treatment response and checking persistence of inflammation [26, 27]. A number of promising alternatives to FDG/PET are being introduced to overcome the limits of current techniques [26]. Whole-body or focused MRI, with or without a contrast agent such as gadolinium, is used to assess hidden areas of active sarcoidosis. MRI does not involve ionizing radiation, but gadolinium contrast agents are contraindicated in patients with severe renal failure (glomerular filtration rate $<30 \mathrm{ml} / \mathrm{min}$ ). 
Newer cardiac implantable devices and pacemakers should permit cardiac magnetic resonance, but can cause artefacts. It is increasingly reported that PET/TC and MRI have a complementary role in the diagnosis and follow-up of cardiac sarcoidosis (CS) [28, 29].

\section{FIBROBRONCHOSCOPY}

Fibrobronchoscopy is increasingly used to perform endobronchial biopsies and transbronchial needle aspiration (TBNA). The addition of endobronchial ultrasound guidance (EBUS) and rapid on-site evaluation (ROSE) of biopsy specimens have improved outcomes with respect to conventional TBNA. The diagnostic yield of EBUS/TBNA for hilar or mediastinal nodes is greater in scadding stages 1 and 2 and ranges from 80 to $90 \%$ [30]. In stage 3 or 4 sarcoidosis, transbronchial lung biopsies have a diagnostic yield of $50-75 \%$ [2]. Endoscopic ultrasound (EUS), usually performed by gastroenterologists, provides access to certain lymph node stations $(2 \mathrm{~L}, 4 \mathrm{~L}, 7,8,9)$ and may be better tolerated than EBUS. Bronchoalveolar lavage (BAL) is not diagnostic for sarcoidosis, although some researchers think that it can be useful to narrow the differential diagnosis. However, some findings such as lymphocytosis (>15\% of total cell count) and a lymphocyte sub-analysis count showing elevated CD4+/ CD8 ratio (a ratio $>3.5$ increases the specificity of the finding) suggest sarcoidosis [31, 32].

\section{PULMONARY FUNCTION AND EXERCISE TESTS}

Pulmonary function tests (PFTs) can initially be normal or show different patterns of alterations depending on lung involvement [2]. Possible restriction is more typical, but obstruction is also common. Airflow obstruction was observed in almost $25 \%$ of a UK cohort of patients with sarcoidosis [20]. The finding and the degree of alterations in PFT are often unrelated to respiratory or general symptoms. The six-minute walk test (6MWT) is commonly performed, as it provides an objective measurement of exercise capacity [33].

\section{SERUM TESTS}

Serum examinations can help narrow the differential diagnosis and/or suggest otherwise asymptomatic organ involvement in sarcoidosis. Serum angiotensin-converting enzyme (ACE) is the most widely used biochemical marker in sarcoidosis. The sensitivity of ACE is rather good, but an elevated level alone is not specific enough to permit diagnosis [34]. Moreover, some genetic backgrounds and concomitant treatments with ACE-inhibitor drugs can reduce its significance. Other serum biomarkers, such as chitotriosidase, interleukin2 receptor (IL-2R), Krebs von den Lungen-6 (KL6) and serum amyloid (SAA), though not always available in practice [35], could be markers of extrathoracic involvement or have prognostic value, but more work is needed to confirm their utility [34]. The role of genetics is increasingly recognized as very important in sarcoidosis, but there is currently no routine clinical application $[6,34]$.

Although relatively uncommon, eye, nervous system, and heart involvement in sarcoidosis are very important as they expose the patient to potentially high morbidity and mortality. If such involvement is suspected, the patients should be referred to an ophthalmologist, cardiologist, or neurologist.

\section{OCULAR SARCOIDOSIS}

Ocular sarcoidosis (OS) is relatively common and can lead to severe irreversible visual impairment. Although any part of the eye may be involved (including adnexa), uveitis, usually bilateral, anterior and chronic, is the most typical lesion. Symptoms generally include eye pain, redness, photophobia, and lacrimation but, up to one-third of patients with anterior uveitis do not report any symptom. Posterior uveitis may also lead to visual impairment with subacute development. As OS may have a smouldering course, ophthalmological 
examination is often suggested in the routine work-up of sarcoidosis. Likewise, OS may be the first manifestation of disease [14]. As sarcoidosis is relatively frequent, ophthalmologists faced with uveitis of unknown origin may prescribe assay of serum ACE and chest CT to exclude or at least reduce the possibility [36]. In a population of 1325 patients with unselected uveitis, $4.15 \%$ had or were presumed to have OS [37]. Diagnostic criteria for OC [38] identify seven clinical ocular signs and three levels of confidence for diagnosis: definite (supported by biopsy), presumed, and probable.

\section{NEUROSARCOIDOSIS}

Neurosarcoidosis (NS) can be the first manifestation of the disease [9] and may involve the central nervous system (CNS) or peripheral nervous system (PNS) or both. Proposed diagnostic criteria establish definite (including neurological biopsy confirmation), probable (including non-neurological biopsy confirmation), and possible NS [39]. CNS involvement may be associated with a number of clinical events, such as seizures, stroke-like episodes, and sensory and/or motor function alterations; unexplained neuropsychiatric episodes may also suggest CNS involvement in sarcoidosis patients. Cerebrospinal fluid analysis is not specific for NS and usually shows evidence of inflammation with a lymphocytic pattern, high protein levels, and negative cultures. When CNS involvement is suspected, brain and/or cervical, thoracic, and lumbar MRI is indicated, with or without gadolinium, depending on the clinical presentation. MRI is the elective examination for the diagnosis of NS, as intense physiological uptake in the brain may limit the value of FDG/PET. However, although some MRI findings can be rather typical $[40,41]$, they alone are not sufficient for diagnosis and need to be part of a context of other compatible clinical, imaging, and laboratory data, while other causes are ruled out. Cranial neuropathy is the most common manifestation of PNS involvement, sometimes causing unilateral or bilateral facial palsy. Patients with suspected PNS involvement should undergo electromyogram and nerve conduction study [39]. Small fiber neuropathy (SFN) is increasingly recognized and develops in up to $30 \%$ of sarcoidosis patients [42]. SFN manifests with pain, burning, and paraesthesia, often beginning in the feet; dysautonomia may also be observed. SFN has been associated with loss of small fibers; skin biopsy and nerve fibre count have been proposed for diagnosis of SFN [42, 43]; corneal confocal microscopy may be more accurate for evaluating SFN [44], but it is not yet widely available. Other more accessible examinations need to be introduced to clarify the frequency ad role of SFN in sarcoidosis.

\section{CARDIAC SARCOIDOSIS}

Cardiac sarcoidosis (CS) is increasingly reported, presumably due to improvements in diagnostic methods rather than a true increase in incidence. Its prevalence shows ethnic differences, being particularly common in the Japanese population [45]. A multicentre European study found a higher prevalence of CS in patients with ocular $(8.9 \%$ vs. $2.7 \%)$, neurological $(11.4 \%$ vs. $2.8 \%)$, and skin lesions (6.1\% vs. $2,5 \%)$ than in those with no organ involvement [13]. Isolated CS is infrequent [46]. It can be the first manifestation of sarcoidosis. CS is associated with a number of clinical findings and severities, ranging from incidental asymptomatic discovery to sudden death or heart failure. When sarcoid granulomas infiltrate the conducting system, early arrhythmias and/or heart block may occur. Myocardial involvement can lead to heart failure with granulomas rapidly progressing to scar tissue [45]. There is no consensus on the routine examinations for excluding CS in sarcoidosis patients with no clinical evidence of CS; recent blue journal guidelines only recommend electrocardiogram (ECG) [19]. Other traditional examinations, such as transthoracic echocardiogram and Holter monitoring, and cardiac serum biomarkers such as pro-BNP or troponin have rather poor predictive value in baseline screening, while advanced imaging methods have recently gained popularity. Indications for advanced imaging when CS is suspected are listed in Table 4 
Table 4 Indications for advanced imaging in patients suspected of having cardiac sarcoidosis

\section{Diagnosis of extracardiac sarcoidosis AND}

Abnormal ECG defined as complete bundle branch block and/or presence of unexplained pathological Q waves in 2 or more leads

Unexplained abnormal echocardiography defined as a regional wall motion abnormalities and/or wall aneurism and/or basal septum thinning and/or left ventricular ejection fraction $<50 \%$

Abnormal Holter trace defined as sustained or nonsustained ventricular tachycardia

Unexplained cardiac symptoms (syncope, palpitations)

AND reasonable exclusion of other causes of cardiac manifestations

Suspected relapse in patients with a history of CS

Treatment monitoring in patients with CS

Prognostic assessment that may influence therapeutic management

$C S$ cardiac sarcoidosis, $E C G$ electrocardiogram

$[19,25,27-29,45,47-49]$. Cardiac magnetic resonance (CMR) is now the gold standard for ruling out CS at baseline: T2-weighted cardiac images show areas with oedema, typical of inflammation, while late gadolinium enhancement (LGE) is absent in areas of damaged tissue, such as scars $[20,47]$.

Traditionally, a definite diagnosis of CS required confirmation by myocardial biopsy. The sensitivity of endomyocardial biopsies is low $(<25 \%)$, as sarcoidosis granulomas are usually patchy [50]. Advanced imaging techniques have improved our knowledge of CS. In 2017, the guidelines were revised and the major criteria for diagnosis of CS in the absence of a positive endomyocardial biopsy were updated (see Table 5) $[45,47,48]$. From a practical point of view, when extracardiac sarcoidosis is diagnosed and cardiac involvement is suspected, CMR should be performed to obtain diagnostic and prognostic information.
Table 5 Expert consensus criteria for diagnosis of cardiac sarcoidosis (CS)

\section{Diagnosis of extracardiac sarcoidosis AND}

One or more of the following major characteristics:

Unexplained high-degree AVB or fatal ventricular arrhythmia

Unexplained basal thinning of ventricular septum or abnormal ventricular wall anatomy

Unexplained LVEF $<50 \%$

CMR evidence of LGE with a pattern consistent with CS

Abnormally high uptake during radiological imaging

(67 Ga or 18FDG PET/TC) with a pattern consistent with CS

AND reasonable exclusion of other causes of the cardiac manifestations

$C S$ cardiac sarcoidosis, $A V B$ atrioventricular block, $L G E$ late gadolinium enhancement, $L V E F$ left ventricular ejection fraction, $C M R$ cardiac magnetic resonance imaging, $P E T$ positron emission tomography, $67 \mathrm{Ga}$ gallium scintigraphy, $18 F D G P E T / C T$ positron emission tomography with ${ }^{18} \mathrm{~F}$-labelled fluorodeoxyglucose/computed tomography

\section{MAIN COMORBIDITIES AND COMPLICATIONS OF SARCOIDOSIS}

Increased risk of infections, sometimes with opportunist microorganisms, has been observed in patients with sarcoidosis. [51, 52]. Infections are more common in fibrocystic forms with bronchiectasis. This could be related to the use of immunosuppressant drugs and the disease itself [53]. Hemoptysis is relatively frequent, often associated with mycetoma. Pulmonary aspergillosis is a severe complication and a marker of advanced lung disease [54].

Sarcoidosis-associated pulmonary hypertension (SAPH) is another complication of sarcoidosis. The occurrence of SAPH ranges from 5 to $15 \%$ of cases in tertiary referral centres, and it is associated with a significantly worse 
prognosis [2]. The median time between diagnosis of sarcoidosis and diagnosis of SAPH is reported to be 17 years, meaning that it is usually a late complication [55]. SAPH should be suspected when there is a disproportionate increase in exertional dyspnoea or alteration in DLco or $6 \mathrm{MWT}$ distance in relation to lung involvement. Echocardiography is the first baseline examination required for confirmation, supplemented, if necessary, with cardiac catheterisation.

On the whole, comorbidities have been found to be more common in sarcoidosis patients than in healthy controls, and have a negative impact on quality of life and prognosis [56]. Sarcoidosis has been associated with a range of comorbidities, including cardiovascular, thromboembolic, autoimmune, and sleeprelated respiratory diseases, diabetes, osteoporosis, and cancer [57-60]. Major depressive and cognitive disorders, including memory loss and concentration difficulty [61], are also frequently reported and may be related to systemic spillage of inflammatory mediators.

\section{MONITORING THE COURSE OF SARCOIDOSIS AND RESPONSE TO THERAPY}

As sarcoidosis is a chronic disease with a highly variable course, periodic checks are necessary [62]. Regarding definitions used during followup, remission usually indicates disappearance of symptoms and/or radiological lesions; relapse is the reappearance of the disease either during the tapering phase or up to 1 year after discontinuation of treatment; recurrence is reappearance of the disease after at least one year of remission; end-stage is irreversible organ damage, unlikely to respond to immunosuppressants or to resolve on its own [12]. Each checkup should mimic the initial work-up (see Fig. 1), with additional monitoring of treatment-related toxicity [63]. Although most extrapulmonary organ involvement occurs at baseline or within 6 months of diagnosis, some organs can subsequently become affected or reveal their involvement, so that extensive longitudinal monitoring needs to be maintained. In the
ACCESS study [10], new organ involvement was reported to occur in $23 \%$ of sarcoidosis patients at a 2-year follow-up.

Close monitoring of OS, NS, and CS is very important, as irreversible eye, cardiac, and neurological lesions may develop rapidly.

Regarding OC, in addition to periodic ophthalmological assessments, enhanced-depth imaging optical coherence tomography and/or indocyanine green angiography are increasingly used to check the course of choroidal sarcoidosis granulomas [64].

Regarding CS, while generally useful and sensitive for baseline diagnosis, CMR is less useful for guiding immunosuppressant therapy. By contrast, FDG-PET is very useful for measuring response to therapy. It has been suggested that patients with CS should be assessed monthly during active pharmacological treatment to confirm suppression of inflammation, and during tapering until cessation. At this point, if imaging and clinical examinations continue to be reassuring, additional PET/CT scans can be performed if relapse is suspected $[49,65,66]$. Echocardiography is also useful for monitoring CS, for studying left ventricular ejection fraction (LVEF), which has strong prognostic significance, and for detecting SAPH, a dangerous late complication of sarcoidosis $[45,67]$. However, there is a continuing lack of reliable and cost-effective markers of extrapulmonary sarcoidosis, especially OC, CS, and NS.

Monitoring of pulmonary sarcoidosis is better defined than that of extrapulmonary forms. Although flares are always possible [51, 68], progression in lung sarcoidosis is usually slow. Among the results of lung function tests, forced vital capacity (FVC) is an important parameter for monitoring disease (and response to treatment) in pulmonary sarcoidosis; the diffusing capacity of the lung for carbon monoxide (DLCo) and forced expiratory volume in the first second (FEV1) are also important [69]. A Delphi panel agreed that changes in PFT during followup are more important than the absolute values of individual tests. A significant reduction in FVC and/or DLco believed to be associated with sarcoidosis could be an indication for a review of therapy [69]. Many authors add periodic measurement of 6MWT, which is also of 
prognostic significance [70]. Although there is no consensus on follow-up times, an assessment at least every 3-6 months in the first 2 years has been proposed, and thereafter yearly for the next $3-5$ years, after which no more follow-up is necessary unless recurrence or new symptoms occur. Imaging is also important for monitoring disease course [23], and is correlated with changes in lung function parameters [71], but no monitoring calendar (or when more advanced examinations should be performed) has been defined. A number of questionnaires have been developed specifically to evaluate symptom burden in sarcoidosis, including the Sarcoidosis Health Questionnaire [72], King's Sarcoidosis Questionnaire [73], Sarcoidosis Assessment Tool [74], and Fatigue Assessment Scale [75]. Some composite scores have been introduced for guiding treatment and monitoring of pulmonary sarcoidosis. The Sarcoidosis Treatment Score includes measurements of FVC ( $\pm 5 \%$ absolute change in baseline value) and DLco changes, high-resolution CT variations, two quality-of-life questionnaires (the King's Sarcoidosis Questionnaire and the Fatigue Assessment Scale), and variations in daily dosage of glucocorticoid, the most commonly used drug for treating sarcoidosis [76]. Another outcome set adds some serum biomarkers, weight gain, and assessment of osteoporosis every 3-6 months, depending on the severity of the disease course [77]. Genetic studies are promising and could be associated with some phenotypes and disease courses, but they are not yet available for routine application. Hopefully, more effective markers will soon be found for progression towards chronic fibrosis, extrapulmonary involvement, prediction of relapse, complications, and therapeutic response [32, 78].

\section{CONCLUSION}

Sarcoidosis has highly variable clinical manifestations, many of which are shared with other, more common diseases. The pulmonologist usually has a key role in the diagnosis and management of sarcoidosis, because the lungs are the organ most commonly affected.
However, a multidisciplinary approach is suggested to achieve the best outcome, as involvement of extrapulmonary organs is not uncommon and is often clinically significant. Diagnosis is often challenging; the diagnosis of sarcoidosis is based on probability, and alternative possibilities should always be borne in mind. Once diagnosis has been made or is suspected, an extensive work-up is required to establish organ involvement. Then, close and detailed follow-up is required, as the natural history of sarcoidosis is unpredictable. An important goal is finding reliable predictors for establishing prognosis and treatment at the individual level [74].

\section{ACKNOWLEDGEMENTS}

Funding. No funding or sponsorship was received for this study or publication of this article.

Authorship. All named authors meet the International Committee of Medical Journal Editors (ICMJE) criteria for authorship for this article, take responsibility for the integrity of the work as a whole, and have given their approval for this version to be published

Authors' contribution. All authors contributed equally to the final paper, including study conception and design, review of the searched literature, and drafting of the manuscript. All authors contributed to the revision and approved the final version.

Disclosure. Andrea S. Melani, Albano Simona, Martina Armati, Miriana d'Alessandro, and Elena Bargagli have nothing to disclose.

Compliance with Ethics Guidelines. This article is based on previously conducted studies and does not contain any new studies with human participants or animals performed by any of the authors.

Open Access. This article is licensed under a Creative Commons Attribution-NonCommercial 
4.0 International License, which permits any noncommercial use, sharing, adaptation, distribution and reproduction in any medium or format, as long as you give appropriate credit to the original author(s) and the source, provide a link to the Creative Commons licence, and indicate if changes were made. The images or other third party material in this article are included in the article's Creative Commons licence, unless indicated otherwise in a credit line to the material. If material is not included in the article's Creative Commons licence and your intended use is not permitted by statutory regulation or exceeds the permitted use, you will need to obtain permission directly from the copyright holder. To view a copy of this licence, visit http://creativecommons.org/ licenses/by-nc/4.0/.

\section{REFERENCES}

1. Brito-Zerón P, Kostov B, Superville D, Baughman RP, Ramos-Casals M, Autoimmune Big Data Study Group. Geoepidemiological big data approach to sarcoidosis: geographical and ethnic determinants. Clin Exp Rheumatol. 2019;37(6):1052-64.

2. Spagnolo P, Rossi G, Trisolini R, Sverzellati N, Baughman RP, Wells AU. Pulmonary sarcoidosis. Lancet Respir Med. 2018;6(5):389-402.

3. Duchemann B, Annesi-Maesano I, Jacobe NC, et al. Prevalence and incidence of interstitial lung diseases in a multi-ethnic county of Greater Paris. Eur Respir J. 2017;50(2):1602419. https://doi.org/10. 1183/13993003.02419-2016.

4. Prior C, Knight RA, Herold M. Pulmonary sarcoidosis: patterns of cytokine release in vitro. Eur Respir J. 1996;9:47-55.

5. Cinetto F, Agostini C. Advances in understanding the immunopathology of sarcoidosis and implications on therapy. Exp Rev Clin Immunol. 2016;12(9):973-88.

6. Miedema JR, Kaiser Y, Broos CE, Wijsenbeek MS, Grunewald J, Kool M. Th17-lineage cells in pulmonary sarcoidosis and Lofgren's syndrome: Friend or foe? J Autoimmun. 2018;87:82-96. https://doi. org/10.1016/j.jaut.2017.12.012 ((Epub 2018 Jan 5. PMID: 29310925)).

7. Newman LS, Rose CS, Bresnitz EA, et al. A case control etiologic study of sarcoidosis: environmental and occupational risk factors. Am J Respir Crit Care Med. 2004;170(12):1324-30.

8. Gupta D, Agarwal R, Aggarwal AN, et al. Molecular evidence for the role of mycobacteria in sarcoidosis: a meta-analysis. Eur Respir J. 2007;30(3):508-16.

9. Llanos O, Hamzeh N. Sarcoidosis. Med Clin North Am. 2019;103(3):527-34. https://doi.org/10.1016/j. mcna.2018.12.011 (Epub 2019 Feb 21 PMID: 30955519).

10. Baughman RP, Teirstein AS, Judson MA, et al. A Case Control Etiologic Study of Sarcoidosis (ACCESS) Research Group. Clinical characteristics of patients in a case control study of sarcoidosis. Am J Respir Crit Care Med. 2001;164(10 Pt 1): 1885-9.

11. Judson MA, Boan AD, Lackland DT. The clinical course of sarcoidosis presentation, diagnosis and treatment in a large white and black cohort in the United States. Sarcoidosis Vasc Diffuse Lung Dis. 2012;29(2):119-27.

12. Mañá J, Rubio-Rivas M, Villalba N, et al. Multidisciplinary approach and long-term follow-up in a series of 640 consecutive patients with sarcoidosis: Cohort study of a 40-year clinical experience at a tertiary referral center in Barcelona, Spain. Medicine (Baltimore). 2017;96(29):7595.

13. Schupp JC, Freitag-Wolf S, Bargagli E, et al. Phenotypes of organ involvement in sarcoidosis. Eur Respir J. 2019;69:77-85.

14. James WE, Koutroumpakis E, Saha B, Nathani A, Saavedra L, Yucel RM, Judson MA. Clinical features of extrapulmonary sarcoidosis without lung involvement. Chest. 2018;154(2):349-56.

15. Bahmer T, Watz H, Develaska $M$, et al. Physical activity and fatigue in patients with sarcoidosis. Respiration. 2018;95(1):18-26.

16. Cho PSP, Vasudevan S, Maddocks M, Spinou A, Chamberlain Mitchell S, Wood C, Jolley CJ, Birring SS. Physical inactivity in pulmonary sarcoidosis. Lung. 2019;197(3):285-93.

17. Hunninghake GW, Costabel U, Ando M, et al. ATS/ ERS/WASOG statement on sarcoidosis. American Thoracic Society/European Respiratory Society/world Association of Sarcoidosis and other granulomatous disorders. Sarcoidosis Vasc Diffuse Lung Dis. 1999;16(2):149-73.

18. Judson MA, Costabel U, Drent $M$, et al. Organ Assessment Instrument Investigators, The Wasog Sarcoidosis. The WASOG Sarcoidosis Organ Assessment Instrument: an update of a previous clinical 
tool. Sarcoidosis Vasc Diffuse Lung Dis. 2014;31(1): 19-27.

19. Crouser ED, Maier LA, Wilson KC, et al. on behalf of the American Thoracic Society Assembly on Clinical Problems. Diagn Detect Sarcoidosis. 2020;201(8):e26-51. https://doi.org/10.1164/rccm. 202002-0251ST (PMID: 32293205).

20. Thillai M, Chang W, Chaudhuri N, et al. Sarcoidosis in the UK: insights from British Thoracic Society registry data. BMJ Open Respir Res. 2019;6(1): e000357. https://doi.org/10.1136/bmjresp-2018000357.

21. Walsh SL, Wells AU, Sverzellati N, et al. An integrated clinicoradiological staging system for pulmonary sarcoidosis: a case-cohort study. Lancet Respir Med. 2014;2(2):123-30.

22. Judson MA. The three tiers of screening for sarcoidosis organ involvement. Respir Med. 2016;113: 42-9.

23. Scadding JG. Prognosis of intrathoracic sarcoidosis in England. A review of 136 cases after five years' observation. Br Med J. 1961;2(5261):1165-72.

24. Reddy GP, Ahuia J. Thoracic sarcoidosis: imaging patterns. Sem Roentgenol. 2019;54(1):59-65.

25. Slart RHJA, Glaudemans AWJM, Lancellotti P, et al. A joint procedural position statement on imaging in cardiac sarcoidosis: from the Cardiovascular and Inflammation \& Infection Committees of the European Association of Nuclear Medicine, the European Association of Cardiovascular Imaging, and the American Society of Nuclear Cardiology. Eur Heart J Cardiovasc Imaging. 2017;18:1073-89.

26. Piekarski E, Benali K, Rouzet F. Nuclear imaging in sarcoidosis. Semin Nucl Med. 2018;48(3):246-60

27. Keijsers RGM, Grutters JC. In Which Patients with Sarcoidosis Is FDG PET/CT Indicated? J Clin Med. 2020;9(3):890. https://doi.org/10.3390/ jcm9030890.

28. Vita T, Okada DR, Veillet-Chowdhury $M$, et al. Complementary value of cardiac Magnetic Resonance Imaging and Positron Emission Tomography/Computed Tomography in the assessment of cardiac sarcoidosis. Circ Cardiovasc Imaging. 2018;11(1):e007030.

29. Miller EJ, Culver DA. Establishing an evidencebased method to diagnose cardiac sarcoidosis: the complementary use of cardiac Magnetic Resonance Imaging and FDG-PET. Circ Cardiovasc Imaging. 2018;11(1):e00740.
30. Trisolini R, Baughman RP, Spagnolo P, Culver DA. Endobronchial ultrasound-guided transbronchial needle aspiration in sarcoidosis: beyond the diagnostic yield. Respirology. 2019;24(6):531-42.

31. Meyer KC, Raghu G, Baughman RP, et al. An Official American Thoracic Society clinical practice guideline: the clinical utility of bronchoalveolar lavage cellular analysis in interstitial lung disease. Am J Respir Crit Care Med. 2012;185(12):1004-14.

32. d'Alessandro $\mathrm{M}$, Carleo A, Cameli $\mathrm{P}$, et al. BAL biomarkers' panel for differential diagnosis of interstitial lung diseases. Clin Exp Med. 2020. https://doi.org/10.1007/s10238-020-00608-5.

33. Baughman RP, Sparkman BK, Lower EE. Six-minute walk test and health status assessment in sarcoidosis. Chest. 2007;132:207-13.

34. Chopra A, Kalkanis A, Judson MA. Biomarkers in sarcoidosis. Exp Rev Clin Immunol. 2016;12(11): 1191-208.

35. Kampstra NA, van der Nat PB, Dijksman LM, et al. Results of the standard set for pulmonary sarcoidosis: feasibility and multicentre outcomes. ERJ Open Res. 2019 Oct 30;5(4):00094-2019. https:// doi.org/10.1183/23120541.00094-2019.

36. El Ameen AE, Herbort CP Jr. Comparison of retinal and choroidal involvement in sarcoidosis-related chorioretinitis using fluorescein and indocyanine green angiography. J Ophtalmic Vis Res. 2018;13: 426-32.

37. Han YS, Rivera-Grana E, Salek S, Rosenbaum JT. Distinguishing uveitis secondary to sarcoidosis from idiopathic disease: cardiac implications. JAMA Ophthalmol. 2018;136(2):109-15.

38. Mochizuki M, Smith JR, Takase H, Kaburaki T, Acharaya NR, Rao NA, for the International Workshop on Ocular Sarcoidosis Study Group. Revised criteria International Workshop on Ocular Sarcoidosis (IWOS) for the diagnosis of ocular sarcoidosis. Br J Ophthamol 2019; 103(10):1418-1422

39. Stern BJ, Royal W 3rd, Gelfand JM, et al. Definition and consensus diagnostic criteria for neurosarcoidosis: from the Neurosarcoidosis Consortium Consensus Group. JAMA Neurol. 2018;75(12): 1546-53.

40. Bathla G, Freeman CW, Moritani T, Song JW, Srivastava S, Soni N, Derdeyn C, Mohan S. Retrospective, dual-centre review of imaging findings in neurosarcoidosis at presentation: prevalence and imaging sub-types. Clin Radiol. 2020;75(10):796.e1796.e9. https://doi.org/10.1016/j.crad.2020.05.008. 
41. Saygin D, Jones S, Sundaram P, Calabrese LH, Messner W, Tavee JO, Hajj-Ali RA. Differentiation between neurosarcoidosis and primary central nervous system vasculitis based on demographic, cerebrospinal and imaging features. Clin Exp Rheumatol. 2020;124(2):135-8.

42. Tavee J, Culver D. Sarcoidosis and small-fiber neuropathy. Curr Pain Headache Rep. 2011;15(3): 201-6.

43. Basantsova NY, Starshinova AA, Dori A, Zinchenko YS, Yablonskiy PK, Shoenfeld Y. Small-fiber neuropathy definition, diagnosis, and treatment. Neurol Sci. 2019;40(7):1343-2135.

44. Brines M, Culver DA, Ferdousi M, Tannemaat MR, van Velzen M, Dahan A, Malik RA. Corneal nerve fiber size adds utility to the diagnosis and assessment of therapeutic response in patients with small fiber neuropathy. Sci Rep. 2018;8(1):4734.

45. Birnie DH, Nery PB, Ha AC, Beanlands RS. Cardiac sarcoidosis. J Am Coll Cardiol. 2016;68(4):411-21.

46. Juneau D, Nery P, Russo J, de Kemp RA, Leung E, Beanlands RSB, Birnie DH. How common is isolated cardiac sarcoidosis? Extra-cardiac and cardiac findings on clinical examination and whole-body ${ }^{18} \mathrm{~F}$ fluorodeoxyglucose positron emission tomography. Int J Cardiol. 2018;253:189-93.

47. Birnie DH, Sauer WH, Bogun F, et al. HRS expert consensus statement on the diagnosis and management of arrhythmias associated with cardiac sarcoidosis. Heart Rhythm. 2014;11(7):1305-12.

48. Terasaki F, Yoshinaga K. New guidelines for diagnosis of cardiac sarcoidosis in Japan. Ann Nucl Cardiol. 2017;3:42-5.

49. Chareonthaitawee P, Beanlands RS, Chen W, on behalf of the group, et al. Joint SNMMI-ASNC Expert Consensus Document on the role of $18 \mathrm{~F}$ FDG PET/CT in cardiac sarcoid detection and therapy monitoring. J Nucl Med. 2017;58(8):1341-53.

50. Khan T, Selvakumar D, Trivedi S, Rao K, Harapoz M, Thiagalingam A, Denniss AR, Varikatt W. The value of endomyocardial biopsy in diagnosis and guiding therapy. Pathology. 2017;49(7):750-6.

51. Baughman RP, Lower EE. Frequency of acute worsening events in fibrotic pulmonary sarcoidosis patients. Respir Med. 2013;107(12):2009-13.

52. Duréault A, Chapelon C, Biard L, et al. Severe infections in sarcoidosis: Incidence, predictors and long-term outcome in a cohort of 585 patients. Medicine (Baltimore). 2017;96(49):e8846.
53. Sweiss NJ, Salloum R, Gandhi S, et al. Significant CD4, CD8, and CD19 lymphopenia in peripheral blood of sarcoidosis patients correlates with severe disease manifestations. PLoS ONE. 2010;5(2):e9088.

54. Uzunhan Y, Nunes H, Jeny F, et al. Chronic pulmonary aspergillosis complicating sarcoidosis. Eur Respir J. 2017;49(6):1602396. https://doi.org/10. 1183/13993003.02396-201.

55. Boucly A, Cottin V, Nunes H, et al. Management and long-term outcomes of sarcoidosis-associated pulmonary hypertension. Eur Respir J. 2017;50(4): 1700465. https://doi.org/10.1183/13993003.004652017.

56. Brito-Zerón P, Acar-Denizli N, Sisó-Almirall A, et al. The burden of comorbidity and complexity in sarcoidosis: impact of associated chronic diseases. Lung. 2018;196(2):239-48.

57. Bours S, de Vries F, van der Bergh JPW, et al. Risk of vertebral and non-vertebral fractures in patients with sarcoidosis: a population-based cohort. Osteoporos Int. 2016;27:1603-10.

58. Ungprasert P, Crowson CS, Matteson EL. Association of sarcoidosis with increased risk of VTE: a population-based study. 1976 to 2013. Chest. 2017;151:425-30.

59. Ungprasert P, Crowson CS, Matteson EL. Risk of cardiovascular disease among patients with sarcoidosis: a population-based retrospective cohort study, 1976-2013. Eur Respir J. 2017;49(2): 1601290. https://doi.org/10.1183/13993003.012902016.

60. Wu C-H, Chung PI, Wu CY, Chen YT, Chiu YW, Chang YT. Liu HN Comorbid autoimmune diseases in patients with sarcoidosis: a nationwide casecontrol study in Taiwan. J Dermatol. 2017;44(4): 423-30.

61. Hendriks C, Drent M, De Kleijn W, Elfferich M, Wijnen P, De Vries J. Everyday cognitive failure and depressive symptoms predict fatigue in sarcoidosis: a prospective follow-up study. Respir Med. 2018;138S:S24-30.

62. Baughman RP, Nagai S, Balter M, et al. Defining the clinical outcome status (COS) in sarcoidosis: results of WASOG Task Force. Sarcoidosis Vasc Diffuse Lung Dis. 2011;28:56-64.

63. Judson MA, Chaudhry H, Louis A, Lee K, Yucel R. The effect of corticosteroids on quality of life in a sarcoidosis clinic: the results of a propensity analysis. Respir Med. 2015;109(4):526-31.

64. Invernizzi A, Agarwal A, Mapelli C, Nguyen QD, Staurenghi G, Viola F. Longitudinal follow-up of 
choroidal granulomas using enhanced depth imaging optical coherence tomography. Retina. 2017;37(1):144-53.

65. Ning N, Guo HH, Iagaru A, Mittra E, Fowler M, Witteles R. Serial cardiac FDG-PET for the diagnosis and therapeutic guidance of patients with cardiac sarcoidosis. J Card Fail. 2019;25(4):307-11.

66. Oakada DR, Saad E, Wand AL, et al. Effect of corticosteroid dose and duration on 18-fluorodeoxyglucose positron Emission Tomography in cardiac sarcoidosis. JACC Cardiovasc Imaging. 2020. https://doi.org/10.1016/j.jcmg.2019.12.013.

67. Kiko T, Yoshihisa A, Kanno Y, et al. A multiple biomarker approach in patients with cardiac sarcoidosis. Int Heart J. 2018;59:996-100.

68. Panselinas E, Judson MA. Acute pulmonary exacerbations of sarcoidosis. Chest. 2012;142:827-36.

69. Rahaghi FF, Baughman RP, Saketkoo LA, et al. Delphi consensus recommendations for a treatment algorithm in pulmonary sarcoidosis. Eur Respir Rev. 2020;29(155):190146. https://doi.org/10.1183/ $16000617.0146-2019$.

70. Shlobin OA, Kouranos V, Barnett SD, et al. Physiological Predictors of Survival in Patients with Sarcoidosis Associated Pulmonary Hypertension: Results from an International Registry. Eur Respir J. 2020. https://doi.org/10.1183/13993003.017472019.

71. Duan J, Xu Y, Zhu H, et al. Relationship between CT activity score with lung function and the serum angiotensin converting enzyme in pulmonary sarcoidosis on chest HRCT. Medicine (Baltimore). 2018;97(36):e12205.

72. Cox CE, Donohue JF, Brown CD, Kataria YP, Judson MA. The Sarcoidosis Health Questionnaire: a new measure of health-related quality of life. Am J Respir Crit Care Med. 2003;168(3):323-9. https:// doi.org/10.1164/rccm.200211-1343OC (Epub 2003 May 8PMID: 12738606).

73. Patel AS, Siegert RJ, Creamer D, et al. The development and validation of the King's Sarcoidosis Questionnaire for the assessment of health status. Thorax. 2013 Jan;68(1):57-65. https://doi.org/10. 1136/thoraxjnl-2012-201962 (Epub 2012 Oct 12PMID: 23065052).

74. Judson MA, Mack M, Beaumont JL, Watt R, Barnathan ES, Victorson DE. Validation and important differences for the Sarcoidosis Assessment Tool. A new patient-reported outcome measure. Am J Respir Crit Care Med. 2015;191(7):786-95. https:// doi.org/10.1164/rccm.201410-1785OC.PMID.

75. de Vries J, Michielsen HJ, van Heck GL, Drent M. Measuring fatigue in sarcoidosis: the Fatigue Assessment Scale (FAS). Br J Health Psychol. 2004;9: 279-91.

76. Baughman RP, Sweiss N, Keijsers R, Birring SS, Shipley R, Saketkoo LA, Lower EE. Repository corticotropin for chronic pulmonary sarcoidosis. Lung. 2017;195(3):313-22.

77. Kampstra NA, Grutters JC, van Beek FT, et al. First patient-centred set of outcomes for pulmonary sarcoidosis: a multicentre initiative. BMJ Open Respir Res. 2019;6(1):000394.

78. Bergantini L, d'Alessandro M, Vietri L, et al. Utility of serological biomarker' panels for diagnostic accuracy of interstitial lung diseases. Immunol Res. 2020;68(6):414-21. https://doi.org/10.1007/ s12026-020-09158-0 (Epub 2020 Oct 22. PMID: 33089426; PMCID: PMC7674352). 